\title{
Lagrangian Mixing Dynamics at the Cloudy-Clear Air Interface
}

\author{
BIPIN KUMAR \\ Max Planck Institute für Meteorologie, Hamburg, Germany \\ JÖRG SCHUMACHER \\ Technische Universität Ilmenau, Ilmenau, Germany \\ RAYMOND A. SHAW \\ Department of Physics, Michigan Technological University, Houghton, Michigan
}

(Manuscript received 5 September 2013, in final form 30 January 2014)

\begin{abstract}
The entrainment of clear air and its subsequent mixing with a filament of cloudy air, as occurs at the edge of a cloud, is studied in three-dimensional direct numerical simulations that combine the Eulerian description of the turbulent velocity, temperature, and vapor fields with a Lagrangian cloud droplet ensemble. Forced and decaying turbulence is considered, such as when the dynamics around the filament is driven by larger-scale eddies or during the final period of the life cycle of a cloud. The microphysical response depicted in $n_{d}-\left\langle r^{3}\right\rangle$ space (where $n_{d}$ and $r$ are droplet number density and radius, respectively) shows characteristics of both homogeneous and inhomogeneous mixing, depending on the Damköhler number. The transition from inhomogeneous to homogeneous mixing leads to an offset of the homogeneous mixing curve to larger dilution fractions. The response of the system is governed by the smaller of the single droplet evaporation time scale and the bulk phase relaxation time scale. Variability within the $n_{d}-\left\langle r^{3}\right\rangle$ space increases with decreasing sample volume, especially during the mixing transients. All of these factors have implications for the interpretation of measurements in clouds. The qualitative mixing behavior changes for forced versus decaying turbulence, with the latter yielding remnant patches of unmixed cloud and stronger fluctuations. Buoyancy due to droplet evaporation is observed to play a minor role in the mixing for the present configuration. Finally, the mixing process leads to the transient formation of a pronounced nearly exponential tail of the probability density function of the Lagrangian supersaturation, and a similar tail emerges in the droplet size distribution under inhomogeneous conditions.
\end{abstract}

\section{Introduction}

The turbulent mixing of cloudy and clear air involves a broad range of spatial and temporal scales, over which water vapor density and temperature fields are coupled to cloud droplet response through evaporation and the associated enthalpy of vaporization. In this work, we study the response of a population of cloud droplets to entrainment and mixing, including the active thermal feedback. Upon the mixing of cloudy and clear air, and

Corresponding author address: Bipin Kumar, Max PlanckInstitut für Meteorologie, Bundesstrasse 53, D-20146 Hamburg, Germany.

E-mail: bipin.kumar@mpimet.mpg.de assuming there is sufficient condensed water in the initial cloud, droplets will evaporate until the mixture becomes saturated. The final, uniquely defined thermodynamic state, however, can be achieved through very different microphysical manifestations. For example, the final, diluted liquid water content (LWC) could be reached in one extreme due to all droplets evaporating by the same amount or in the other extreme due to a subset of droplets evaporating completely, leaving the remaining droplets unchanged. These extremes were first recognized by Latham and Reed (1977) and Baker et al. $(1980,1984)$, who described them as homogeneous and inhomogeneous mixing, respectively. The limits can be conveniently expressed through the Damköhler number, defined as the ratio of a fluid time scale to a characteristic 
thermodynamic time scale associated with the evaporation process:

$$
\mathrm{Da}=\frac{\tau_{\text {fluid }}}{\tau_{\text {phase }}},
$$

with homogeneous and inhomogeneous mixing corresponding to the limits $\mathrm{Da} \ll 1$ and $\mathrm{Da} \gg 1$, respectively (Lehmann et al. 2009; Andrejczuk et al. 2009). Homogeneous mixing occurs when the evaporation of cloud water droplets is slow compared to the mixing and therefore takes place in a well-mixed, or in other words, homogenized environment. Inhomogeneous mixing occurs when the evaporation proceeds much faster than the turbulence evolves, with the result that droplets near the clear air-cloud interface experience evaporation while others do not. Both processes can coexist in a turbulent cloud because of the broad spectrum of fluid time scales that are present, with inhomogeneous mixing dominating at large scales and homogeneous mixing occurring at fine scales (Lehmann et al. 2009).

The characteristic time scale associated with the response of the water vapor density and temperature fields as a result of droplet growth or evaporation is the phase relaxation time $\tau_{\text {phase }}$, which is inversely proportional to the droplet number density and the mean droplet radius (e.g., Kostinski 2009; Kumar et al. 2013). For small droplets or strong dilution by dry air, complete evaporation can occur, suggesting that the appropriate microphysical response time should be the single droplet evaporation time scale $\tau_{\text {evap }}$ (cf. Andrejczuk et al. 2006). It was argued by Lehmann et al. (2009) that the smaller of the two time scales $\tau_{\text {phase }}$ and $\tau_{\text {evap }}$ is the appropriate one for specifying Da and therefore the relative homogeneity of the mixing process, and that suggestion will be further addressed here.

The mixing problem has been approached in many recent studies from the point of view of a mixing diagram showing the microphysical response in terms of cloud droplet mean volume radius $\left\langle r^{3}\right\rangle$ versus number density $n_{d}$. This $n_{d}-\left\langle r^{3}\right\rangle$ space, introduced by Jensen et al. (1985), allows a reduction in liquid water content, $W \propto n_{d}\left\langle r^{3}\right\rangle$, to be interpreted as the relative reductions in cloud droplet number density (through both dilution and total droplet evaporation) and mean droplet diameter. Andrejczuk et al. (2006) were the first to show a "trajectory" within a mixing diagram. Note that these trajectories are for averages taken over the entire volume and are for a "bulk" cloud treatment. General consistency with scaling laws was found. For example, larger droplets gave a more homogeneous mixing signature. In a subsequent paper, Andrejczuk et al. (2009) studied the ratio of time scales in their numerical simulation, in particular the ratio of the turbulence time scale to the droplet evaporation time scale, thereby supporting quantification via the Damköhler number [cf. Eq. (1)]. In a thorough study of measured cloud properties in the mixing diagram, Burnet and Brenguier (2007) showed a distinct predominance for inhomogeneous mixing. They point out that the difficulty in observing homogeneous mixing in clouds may in some cases be a sampling artifact resulting from spatial averaging. Gerber et al. (2008) also detected in their measurements signatures that indicate strong inhomogeneous mixing, but they suggest the possibility that the data could be interpreted as resulting from homogeneous mixing with a small contrast between cloud and environment. The possibility of mixing with already humid air is consistent with the finding of Heus and Jonker (2008), who showed with large-eddy simulations that cumulus clouds are surrounded by subsiding shells in which fluid motion is mostly downward. Thus, the mixing takes place rather locally with diluted cloudy air in the vicinity of the interface rather than with a large-scale environment. These numerical results imply already that homogeneous and inhomogeneous mixing can coexist and are sometimes difficult to disentangle. Finally, Lehmann et al. (2009) gave theoretical arguments and field data in support of the concept that a single Damköhler number is not sufficient to explain the mixing. Based on the cascade concept in turbulence, they also suggest a length scale at which the system transforms from dominantly homogeneous to inhomogeneous mixing.

Kumar et al. (2012) studied extremes of mixing, not via range of scales, but by enhanced or suppressed droplet response within an Euler-Lagrangian model. The studies showed that a Da similarity does exist within the range of idealized conditions studied. In other words, they observed that different turbulent and microphysical initial conditions having the same Da will tend to the same final microphysical state when the mixing process is completed. Kumar et al. (2013) investigated the phase relaxation process during a mixing event under a variety of realistic microphysical conditions for a cumulus cloud. In this Lagrangian view of the mixing process, a range of Da was investigated, and it was shown that the relevant microphysical time scale is the "diluted" phase relaxation time (i.e., calculated with the number density based on the whole volume). In both of these studies, however, the simulations did not include the temperature field and therefore the interaction between latent heat and dynamic coupling through changes in buoyancy.

Besides numerical studies and measurements, a third effort consists of the development of parameterized models describing the essentials of the simultaneous 
mixing at multiple scales. Krueger et al. (1997) and Su et al. (1998) pioneered the extension of the linear eddy model to the entrainment problem, thereby enabling the representation of cloud mixing and microphysical response on multiple length scales without a direct simulation. Such models can be potentially incorporated into cloud physics parameterizations in larger-scale models that do not resolve processes below a few kilometers. In a different approach based on the simulations of Andrejczuk et al. (2006), Grabowski (2007) suggested a simple, subgrid parameterization of cloud droplet responses to bulk mixing based on increasing filamentation of the turbulent eddies in a steady cascade process. Lu et al. (2011) explored the possibility of representing subgrid mixing effects on microphysics via a dimensionless parameter, the scale number, to characterize the dynamics of different entrainment-mixing processes. The scale number relates the transition-scale concept of Lehmann et al. (2009) to the Kolmogorov length, the mean dissipation scale of the turbulence. Lu et al. (2013) have coupled measurements and modeling studies using the linear eddy approach to explore the degree of homogeneous mixing and its dependence on transition scales.

Prior computational investigations of the mixing process, such as those by Jensen and Baker (1989), Andrejczuk et al. (2004, 2006), Malinowski et al. (2008), and de Lozar and Mellado (2014), have considered the problem primarily from the continuum microphysics perspective. De Lozar and Mellado (2014) included some more detailed processes such as droplet sedimentation and particle inertia in their bulk formulation. Here, we explore what additional insight can be gained by explicitly treating the Lagrangian nature of the discrete droplet field, including droplet inertia and gravitational sedimentation. The coupling of Lagrangian droplets to the Eulerian vapor density and temperature fields is similar to the approaches of Vaillancourt et al. (2001, 2002) and Lanotte et al. (2009); in those studies the emphasis was on the evolution of the supersaturation field and the droplet population during steady growth, instead of the microphysical response to a transient mixing event considered here.

In this work, we consider an idealized cloud slab that mixes with a dry environment in a small subvolume at the edge of a cloud. The simple geometry allows for a clearly defined initial scale for the mixing. The initial cloud and environment values are purposely set to rather extreme values, and the motivation for this is explained here. First, the environment is essentially completely dry so that a strong signature of homogeneous mixing can be observed. Observational studies often show rather inhomogeneous signatures, and this can be a result of mixing with very humid air even under homogeneous conditions (e.g., Gerber et al. 2008). Second, the initial cloud is taken to have a range of liquid water content so that cases with partial and with complete evaporation of droplets can be studied. To achieve partial evaporation, very large initial liquid water contents are set in the most extreme case. The goal is to understand the microphysical response to mixing in a wide range of parameter space, as opposed to simulating only "typical" cloud conditions. We will study two different flow scenarios: decaying convective (D) and stationary convective (S) runs. The decaying convective runs study the mixing in a decaying turbulent case with feedback by buoyancy. In the stationary convective runs, the mixing proceeds in a statistically stationary flow sustained by an additional, steady driving force that mimics the impact of larger-scale eddies on the dynamics in the subvolume.

Several consequences of a finescale study, as is conducted here (approximately $0.1 \mathrm{~m}^{3}$ are simulated), should be mentioned in order to place the work in a proper context. Because the subvolume in the present system is rather small, diffusivities of the scalar fields are close to the kinematic viscosity magnitude, and therefore advection and diffusion time scales do not differ by many orders of magnitude. Thus, the mixing process always incorporates both diffusion and stirring (or advection). Both processes cannot be separated from each other for the given parameters, as has been discussed by Broadwell and Breidenthal (1982), Sreenivasan et al. (1989), and Malinowski and Zawadzki (1993). The scale of this study also does not lend itself to defining the simulated mixing process as entrainment versus detrainment; in part because at finescales it is not obvious that a mean cloud surface can be defined, as is required for a formal distinction between the two processes (de Rooy et al. 2013). The focus of the study is on the microphysical response to mixing, regardless of whether it is occurring in entrainment or detrainment regions. Finally, a consequence of the relatively small volume, which can be simulated with existing computational resources, is that the limit of extremely inhomogeneous mixing $(\mathrm{Da} \gg 1)$ is not investigated in this work.

The outline of the manuscript is as follows: The next section describes the Euler-Lagrangian model and the setting of the simulations. Section 3 starts with a brief discussion of the dynamics in both flow settings, followed by a detailed analysis of the mixing diagrams. Furthermore, we compare Lagrangian distributions of the droplet size and supersaturation at droplet positions. We conclude with a summary and an outlook. 


\section{Eulerian-Lagrangian Boussinesq model of mixing}

\section{a. Model equations, parameters, and numerical method}

The buoyancy $B$ is a function of the temperature $T$, the vapor mixing ratio $q_{v}$, and the liquid water mixing ratio $q_{l}$. The latter two quantities are defined as $q_{v}(\mathbf{x}, t)=$ $\rho_{v} / \rho_{d}$ and $q_{l}(\mathbf{x}, t)=\rho_{l} / \rho_{d}$, where $\rho_{v}, \rho_{l}$, and $\rho_{d}$ are the mass densities of vapor, liquid water, and dry air, respectively. The Eulerian equations for the turbulent fields, namely, the velocity field $\mathbf{u}$, the temperature field, and the vapor mixing ratio field, are given by

$$
\begin{gathered}
\nabla \cdot \mathbf{u}=0, \\
\partial_{t} \mathbf{u}+(\mathbf{u} \cdot \nabla) \mathbf{u}=-\frac{1}{\rho_{0}} \nabla p+\nu \nabla^{2} \mathbf{u}+B \mathbf{e}_{z}+\mathbf{f}_{\mathrm{LS}}, \\
\partial_{t} T+\mathbf{u} \cdot \nabla T=\kappa \nabla^{2} T+\frac{L}{c_{p}} C_{d}, \\
\partial_{t} q_{v}+\mathbf{u} \cdot \nabla q_{v}=D \nabla^{2} q_{v}-C_{d} .
\end{gathered}
$$

The reference density $\rho_{0}$ is the dry air density. The buoyancy term in the momentum equation is defined as

$$
B(\mathbf{x}, t)=g\left[\frac{T-T_{0}}{T_{0}}+\tilde{\epsilon}\left(q_{v}-q_{v 0}\right)-q_{l}\right],
$$

where $\tilde{\epsilon}=R_{v} / R_{d}-1 \approx 0.608$. Here, $R_{v}$ is the vapor gas constant, and $R_{d}$ is the dry air gas constant. The additional term $\mathbf{f}_{\mathrm{LS}}$ keeps the flow in a statistically stationary state during the mixing process. It is used to model the driving of the entrainment process resulting from larger scales (LS) that go beyond the volume size considered here. The term is implemented in the Fourier space in the applied pseudospectral method and is given by (see also Kumar et al. 2013)

$$
\mathbf{f}_{\mathrm{LS}}(\mathbf{k}, t)=\epsilon_{\mathrm{in}} \frac{\mathbf{u}(\mathbf{k}, t)}{\sum_{\mathbf{k}_{f} \in \mathcal{K}}\left|\mathbf{u}\left(\mathbf{k}_{f}, t\right)\right|^{2}} \delta_{\mathbf{k}, \mathbf{k}_{f}},
$$

with the Kronecker delta

$$
\delta_{\mathbf{k}, \mathbf{k}_{f}}=1 \quad \text { if } \quad \mathbf{k}=\mathbf{k}_{f}, \quad \delta_{\mathbf{k}, \mathbf{k}_{f}}=0 \quad \text { otherwise },
$$

and the wavevector subset $\mathcal{K}$ that contains a few wavevectors. Here, these wavevectors are given by $\mathbf{k}_{f}=\left(2 \pi / L_{x}\right.$, $\left.2 \pi / L_{y}, 4 \pi / L_{z}\right)$ plus all permutations with respect to components and signs. Physically, we inject a fixed amount of turbulent kinetic energy per time unit into our flow such that, in a statistically stationary regime, the parameter $\epsilon_{\text {in }}$
TABLE 1. Parameters of the initial turbulence conditions for all parameter runs. The root-mean-square velocity is calculated by $u_{\mathrm{rms}}=\left\langle u_{i}^{2}\right\rangle^{1 / 2}$, the Kolmogorov length is calculated by $\eta_{K}=\nu^{3 / 4} /$ $\langle\varepsilon\rangle^{1 / 4}$, and the Kolmogorov time is calculated by $\tau_{\eta}=(\nu /\langle\varepsilon\rangle)^{1 / 2}$.

\begin{tabular}{lll}
\hline \multicolumn{1}{c}{ Quantity } & Symbol & \multicolumn{1}{c}{ Value } \\
\hline Grid points & $N$ & 512 \\
Box length & $L_{x}$ & $51.2 \mathrm{~cm}$ \\
Grid size & $a$ & $1 \mathrm{~mm}$ \\
Kinematic viscosity of air & $\nu$ & $1.5 \times 10^{-5} \mathrm{~m}^{2} \mathrm{~s}^{-1}$ \\
Mean energy dissipation rate & $\langle\varepsilon\rangle$ & $33.75 \mathrm{~cm}^{2} \mathrm{~s}^{-3}$ \\
Kolmogorov length & $\eta_{K}$ & $1 \mathrm{~mm}$ \\
Kolmogorov time & $\tau_{\eta}$ & $0.066 \mathrm{~s}$ \\
Root-mean-square velocity & $u_{\mathrm{rms}}$ & $12.5 \mathrm{~cm} \mathrm{~s}^{-1}$ \\
Large-scale turnover time & $T_{L}$ & $4.1 \mathrm{~s}$ \\
\hline
\end{tabular}

would equal the mean kinetic energy dissipation rate in the flow when the buoyancy feedback would be absent (see also section 3a).

We will denote runs with $\mathbf{f}_{\mathrm{LS}}=0$ as decaying convective runs and $\mathbf{f}_{\mathrm{LS}} \neq 0$ as stationary convective runs. Furthermore, $C_{d}$ is the condensation rate, $\nu$ is the kinematic viscosity of air, $D$ is the vapor mass diffusivity, $\kappa$ is the thermal diffusivity, $c_{p}$ is the specific heat at constant pressure, and $L$ is the latent heat. The problem is studied in a cube (i.e., $L_{x}=L_{y}=L_{z}$ ) with periodic boundary conditions in all three spatial directions. It is spanned by an equidistant mesh with uniform size $a$ equal to the Kolmogorov length $\eta_{K}$. Further parameters of the initial setup are listed in Table 1 . The equations are solved by a standard pseudospectral method that uses three-dimensional fast Fourier transformations (Ferziger and Perić 2001; Spyksma et al. 2006). Time stepping for the Eulerian and Lagrangian parts is done by a second-order predictor-corrector scheme.

The liquid water component is modeled as a Lagrangian ensemble of $N$ pointlike droplets. From this ensemble, the liquid water mixing ratio and the condensation rate are obtained. The droplets are described by

$$
\begin{aligned}
\frac{d \mathbf{X}}{d t} & =\mathbf{V}(\mathbf{X}, t), \\
\frac{d \mathbf{V}}{d t} & =\frac{1}{\tau_{p}}[\mathbf{u}(\mathbf{X}, t)-\mathbf{V}(\mathbf{X}, t)]+\mathbf{g}, \\
r(\mathbf{X}, t) \frac{d r(\mathbf{X}, t)}{d t} & =K S(\mathbf{X}, t) .
\end{aligned}
$$

Here, $\mathbf{X}$ is the droplet position, $\mathbf{V}$ is its velocity, and $r$ is the radius. We describe cloud water droplets as inertial point particles with a finite particle response time $\tau_{p}=$ $2 \rho_{l} r^{2} /\left(9 \rho_{0} \nu\right)$ that can grow and shrink by diffusion of vapor to their surface. The vector $\mathbf{g}=(0,0,-g)$ includes 
(a)

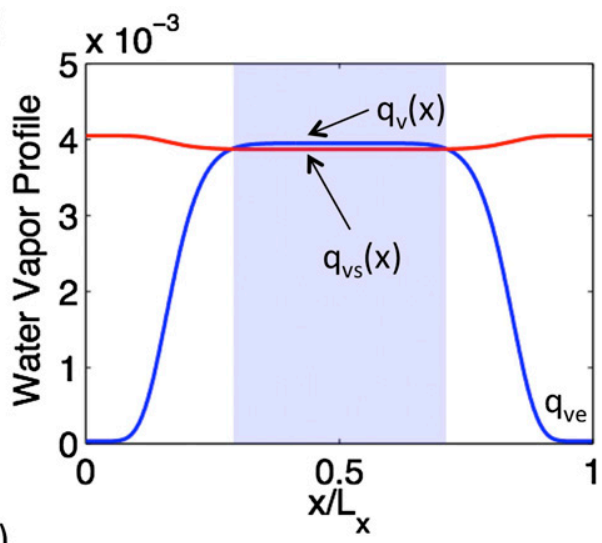

(c)

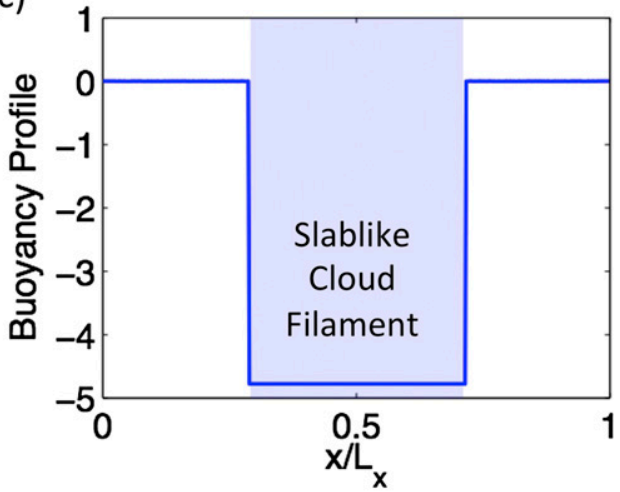

(b)

(d)
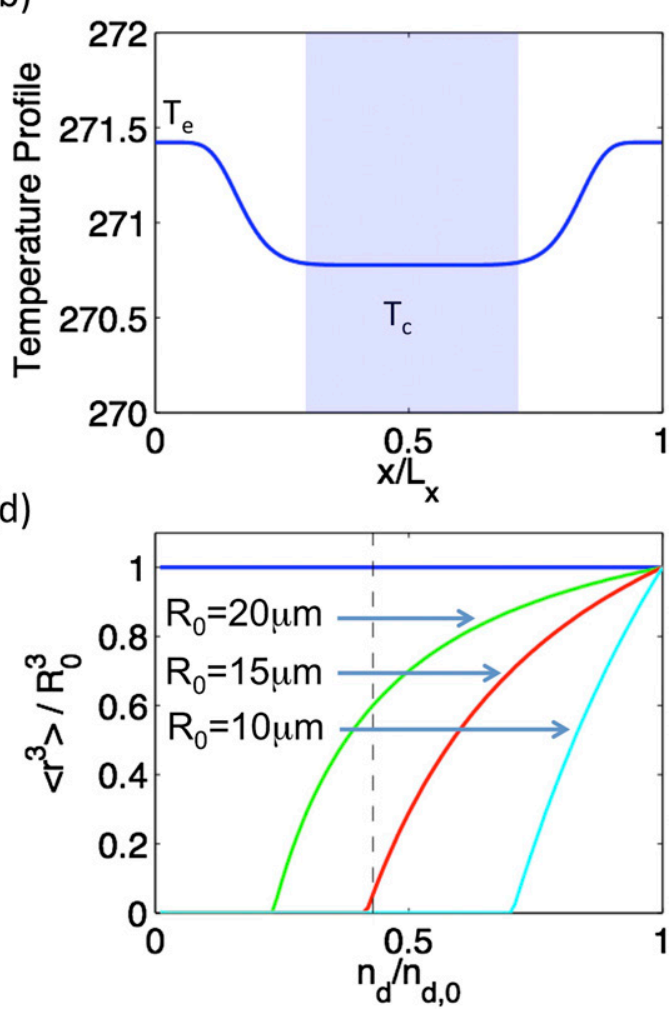

FIG. 1. Initial slablike configuration of the mixing simulations. (a) The water vapor profile $q_{v}(x)$ and $q_{v s}(x)$ that result from the (b) initial temperature profile $T(x)(\mathrm{K})$. The saturation vapor mixing ratio follows from $q_{v s}=e_{s} l$ $\left(R_{v} \rho_{0} T\right)$ and $e_{s}(T)=c_{1} \exp \left(-c_{2} / T\right)$ (Rogers and Yau 1989). In both panels we indicate the supersaturated cloud filament that extends over $L_{x, 1} \leq x \leq L_{x, 2}$ and the whole $y-z$ cross section of the simulation box. Also indicated are the environmental values $q_{v e}$ and $T_{e}$ as well as $T_{c}$. (c) The resulting buoyancy profile $\left(\mathrm{cm} \mathrm{s}^{-2}\right)$, as following from Eq. (6). The profile is obtained for $R_{0}=20 \mu \mathrm{m}$. (d) The inhomogeneous mixing limit (blue horizontal line) and the homogeneous mixing limits for the different initial liquid water contents. The vertical dashed line indicates the volume ratio of subvolume seeded with droplets to the total box volume.

the gravitational acceleration $g$. The constant $K$ in Eq. (11) is a function of temperature and pressure and incorporates the self-limiting effects of latent heat exchange (e.g., Rogers and Yau 1989). This diffusional growth is controlled by the supersaturation, given by $S(\mathbf{X}, t)=q_{v}(\mathbf{X}, t) / q_{v, s}(T)-1$. The saturation vapor mixing ratio $q_{v, s}(T)$ has to be determined from the temperature via the Clausius-Clapeyron equation. For a more detailed derivation of Eq. (11) and its implementation in the simulation, we refer the reader to Kumar et al. (2013). To close the set of equations, we determine the condensation rate field $C_{d}(\mathbf{x}, t)$ following Vaillancourt et al. $(2001,2002)$ by

$$
C_{d}(\mathbf{x}, t)=\frac{4 \pi \rho_{l} K}{\rho_{0} a^{3}} \sum_{\beta=1}^{\Delta} S\left(\mathbf{X}_{\beta}, t\right) r\left(\mathbf{X}_{\beta}, t\right) .
$$

Here, $m_{a}$ is the mass of air per grid cell, and the sum collects the droplets inside each of the grid cells of size $a^{3}$ that surround the (grid) point $\mathbf{x}$. The transmission of the Eulerian field values at grid positions to the enclosed droplet position is done by trilinear interpolation. The inverse procedure is required for the calculation of the condensation rate, which is evaluated at first at the droplet position and then redistributed to the nearest eight grid vertices.

Figures 1a-c show the initial profiles of the fields $q_{v}, T$, and $B$ and indicate the slablike filament of supersaturated vapor in which the monodisperse droplets are seeded homogeneously at the beginning of all runs. Further parameters of the six different simulation runs are summarized in Table 2. We have chosen the initial profiles similar to our previous mixing studies (Kumar et al. 2013). The initial vapor content profile is given by

$$
q_{v}(x, t=0)=\left(q_{v}^{\max }-q_{v e}\right) \exp \left[-\lambda\left(x-\frac{L_{x}}{2}\right)^{8}\right]+q_{v e} .
$$


TABLE 2. Parameters of the six DNS runs. We list the initial droplet radius $R_{0}$, initial liquid water content $W$, the number density in the undiluted initial slablike cloud filament, the phase relaxation time $\tau_{\text {phase }}$, the single droplet evaporation time given by $\tau_{\text {evap }}=-R_{0}^{2} /\left(2 K S_{0}\right)$ [cf. Eq. (15)], the Damköhler numbers based on the Kolmogorov and large-eddy scales, and the Damköhler numbers calculated with the evaporation time scale (rather than the usual phase relaxation time scale). The superscript (0) indicates that the calculations are based on values at the beginning of the simulation $(t=0)$. The large-eddy time for all runs is $T_{L}=4.1 \mathrm{~s}$; the Kolmogorov time is $\tau_{\eta}=0.066 \mathrm{~s}$ ( $\mathrm{see}$ also Table 1). Two scenarios apply here: the purely convective feedback (D1-D3) to the velocity field via the buoyancy term $B$ as given in Eq. (6) or the buoyancy feedback that is combined with an additional volume driving $\mathbf{f}_{\mathrm{LS}}$ beside the buoyancy feedback (S1-S3). The latter mimics the motion of larger turbulent eddies that feed energy into the present subsystem (Schumacher et al. 2007). The slablike cloudy filament is filled with 8.8 million droplets.

\begin{tabular}{lccccccccc}
\hline \hline Case & $R_{0}(\mu \mathrm{m})$ & $W\left(\mathrm{~g} \mathrm{~cm}^{-3}\right)$ & $n_{d}^{(0)}\left(\mathrm{cm}^{-3}\right)$ & $\tau_{\text {phase }}^{(0)}(\mathrm{s})$ & $\tau_{\text {evap }}^{(0)}(\mathrm{s})$ & $\mathrm{Da}_{\eta}^{(0)}$ & $\mathrm{Da}_{L}^{(0)}$ & $\mathrm{Da}_{\eta, \text { evap }}^{(0)}$ & $\mathrm{Da}_{L, \text { evap }}^{(0)}$ \\
\hline S1 & 10 & 0.37 & 153 & 4.12 & 0.93 & 0.016 & 1.0 & 0.07 \\
S2 & 15 & 1.24 & 153 & 2.75 & 2.10 & 0.024 & 1.5 & 0.03 \\
S3 & 20 & 2.95 & 153 & 2.06 & 3.73 & 0.032 & 2.0 & 0.02 \\
D1 & 10 & 0.37 & 153 & 4.12 & 0.93 & 0.016 & 1.0 & 0.07 & 1.1 \\
D2 & 15 & 1.24 & 153 & 2.75 & 2.10 & 0.024 & 1.5 & 0.03 \\
D3 & 20 & 2.95 & 153 & 2.06 & 3.73 & 0.032 & 2.0 & 0.02 \\
\hline
\end{tabular}

Here, $q_{v}^{\max }$ is the maximum amplitude of $q_{v}$, which exceeds $q_{v s}$ by $2 \%$, and $\lambda=1.0 \times 10^{-10} \mathrm{~cm}^{-1}$ is a constant. The initial temperature profile is chosen such that both do not contribute jointly to the initial buoyancy, that is, $T(x, t=0)=T_{0}-\tilde{\epsilon} T_{0}\left[q_{v}(x, t=0)-q_{v 0}\right]$, as derived from Eq. (6). The reference values are given by the volume averages $T_{0}=\langle T(t=0)\rangle_{V}$ and $q_{v 0}=\left\langle q_{v}(t=0)\right\rangle_{V}$. Other initial configurations are possible. Our motivation is to use an initial condition with a well-defined interface that is still a smooth function and avoids the Gibbs phenomenon, that is, numerical overshoots at sharp interfaces.

All runs start with the same turbulent flow field that has been generated in a pure fluid simulation of statistically stationary box turbulence ahead of the entrainment runs. The focus of the study is on the entrainment process, and therefore we neglect droplet collisions. This is physically reasonable given the short mixing times simulated relative to typical droplet collision time scales.

\section{b. Homogeneous and inhomogeneous mixing limits}

The central dimensionless parameter that quantifies the mixing of clear and cloudy air is the Damköhler number defined in Eq. (1). The fluid time scales cover a whole spectrum of values and can vary from the largeeddy turnover time $T_{L}=L_{\mathrm{x}} / u_{\mathrm{rms}}$ to the Kolmogorov time scale $\tau_{\eta}=(\nu /\langle\varepsilon\rangle)^{1 / 2}$. The evaporation process is characterized by the phase relaxation time, given approximately by

$$
\tau_{\text {phase }}=\frac{1}{4 \pi \mathcal{D} n_{d} R_{0}},
$$

where $n_{d}$ is the number density of the droplets, and $\mathcal{D}$ is the vapor diffusion constant that contains the selflimiting effects of latent heat release as described in Kumar et al. (2012). The inhomogeneous limit stands for a very rapid evaporation compared to the evolution time of the fluid. Droplets at the cloud interface will evaporate immediately, while droplets in the center of the cloud remain unaffected by the mixing of the clear air into the cloudy filament. As a consequence, the number density decreases, while the mean cubic radius $\left\langle r^{3}\right\rangle$ remains essentially unchanged. In a mixing diagram showing $\left\langle r^{3}\right\rangle / R_{0}^{3}$ plotted against $n_{d} / n_{d, 0}$, such a process is given by a horizontal line (Jensen et al. 1985; Burnet and Brenguier 2007; Gerber et al. 2008; Lehmann et al. 2009), as shown in Fig. 1d. A second time scale related to the droplet response is the evaporation time given by

$$
\tau_{\text {evap }}=-\frac{r^{2}}{2 K S}
$$

which is a direct consequence of Eq. (11) in the case of a constant supersaturation.

The homogeneous mixing limit assumes a slow microphysical response time scale such that the whole cloud water droplet ensemble evolves in a well-mixed environment. The corresponding mixing lines for the three different initial radii and the parameters of the initial configuration are also indicated in Fig. 1d. The calculation of these lines is performed as follows: it is assumed that precipitation is absent and that the total water content and the liquid water potential temperature, $\theta_{l}=T-\left(L / c_{p}\right) q_{l}$, are state variables that describe the air parcels. Because they are conserved during mixing the following two simple relations hold (e.g., Gerber et al. 2008):

$$
\begin{gathered}
q_{l}+q_{v s}(T)=\chi\left[q_{l c}+q_{v s c}\left(T_{c}\right)\right]+(1-\chi) q_{v e}, \\
T-\frac{L}{c_{p}} q_{l}=\chi\left(T_{c}-\frac{L}{c_{p}} q_{l c}\right)+(1-\chi) T_{e}
\end{gathered}
$$

with (Rogers and Yau 1989) 


$$
q_{v s}(T) \approx \frac{c_{1} \exp \left(-c_{2} / T\right)}{R_{v} \rho_{0} T}
$$

with $c_{1}=2.53 \times 10^{8} \mathrm{kPa}$ and $c_{2}=5420 \mathrm{~K}$. Here, $\chi$ is defined as the mixture fraction; indices $c$ and $e$ stand for cloud and environment, respectively. This nonlinear system of Eqs. (16)-(18) has to be solved by a rootfinding algorithm and results in $q_{l}(\chi)$ and $T(\chi)$ values corresponding to a given $\chi$. The quantity $\left\langle r^{3}\right\rangle$ is obtained via $q_{l}=W / \rho_{0}$ and $W=4 \pi \rho_{l} n_{d, 0} \chi\left\langle r^{3}\right\rangle$. If it is assumed that $\chi=n_{d} / n_{d, 0}$, that is, all droplets respond equally and no subset of droplets is allowed to evaporate completely, then this yields the homogeneous mixing curves in the mixing diagram. The homogeneous mixing process starts from point $(1,1)$ in the upper right and moves from one equilibrium state to another along the mixing lines. Different lines for the homogeneous limit correspond to different initial liquid water content $W$.

\section{Simulation results}

\section{a. Turbulence in decaying and stationary convective regimes}

As already discussed in section 2, all simulations start with a statistically stationary fluid turbulence state corresponding to a Taylor microscale Reynolds number of $R_{\lambda} \approx 90$. We either continue to sustain the driving $\mathbf{f}_{\mathrm{LS}}$, and thus sustain statistical stationarity of the flow field during the mixing event, which is done in the stationary convective regime for runs S1-S3, or we switch off the additional driving, as done in the decaying convective runs D1-D3. In both cases, the feedback from the buoyancy term $B \mathbf{e}_{z}$ is present but turns out to be small. This is likely a result of the relatively low temperature considered in this study. The dynamical evolution is close to freely decaying turbulence for cases D1-D3. Note that we do not sustain a mean temperature gradient with respect to $z$, which would cause a strong buoyancy driving additionally amplified by the periodic boundary conditions (this regime is known as the homogeneous Rayleigh-Bénard convection regime; e.g., Calzavarini et al. 2006).

Figure 2 (top) displays the temporal evolution of the turbulent kinetic energy (TKE). As expected, we observe that the TKE in runs $\mathrm{S} 1-\mathrm{S} 3$ remains on average at the initial value while it decays within a few seconds for runs D1-D3. In the steady convective case,

$$
\begin{aligned}
\langle\varepsilon(t)\rangle_{V} & =\left\langle u_{z} B(t)\right\rangle_{V}+\left\langle\mathbf{u} \cdot \mathbf{f}_{\mathrm{LS}}(t)\right\rangle_{V} \approx\left\langle\mathbf{u} \cdot \mathbf{f}_{\mathrm{LS}}(t)\right\rangle_{V} \\
& \Rightarrow\langle\varepsilon\rangle_{V, t} \approx\left\langle\mathbf{u} \cdot \mathbf{f}_{\mathrm{LS}}(t)\right\rangle_{V, t} .
\end{aligned}
$$

Buoyancy, as the lone driving force in the decaying convective cases, causes a transient growth, but
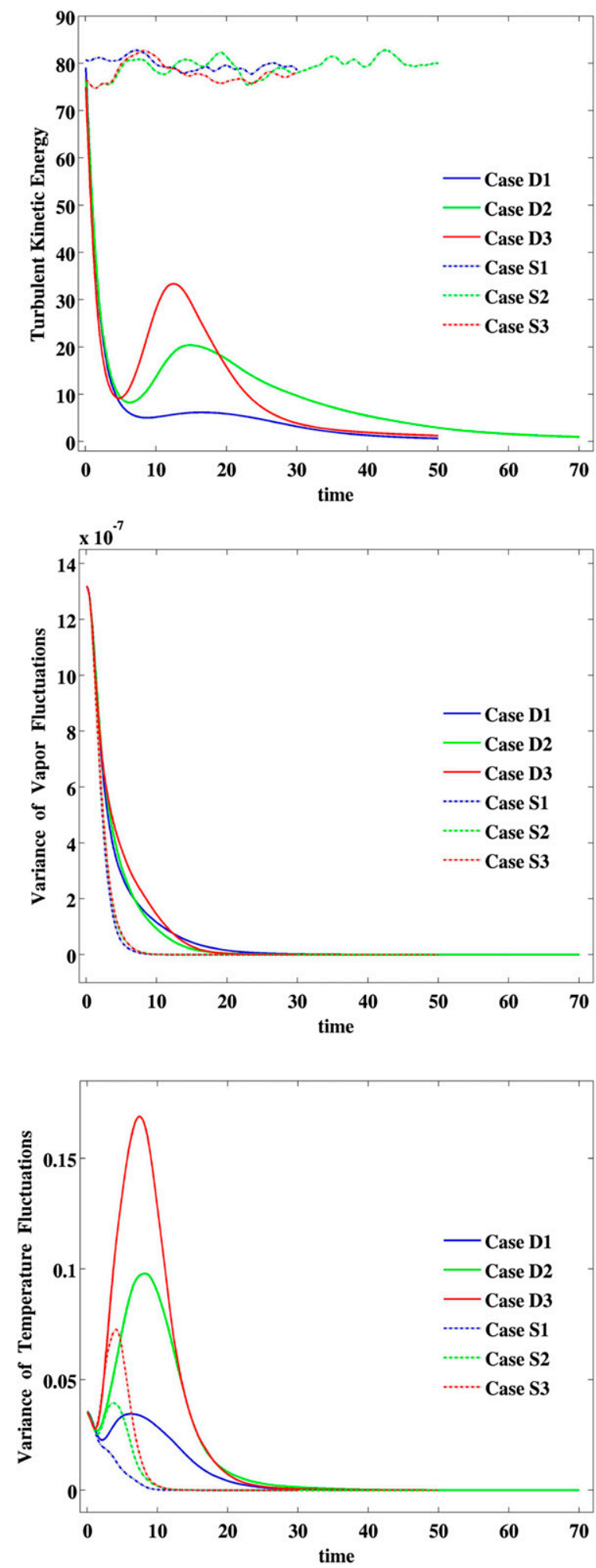

FIG. 2. (top) TKE $\left(\mathrm{cm}^{2} \mathrm{~s}^{-2}\right)$ vs time (s) for all six runs as indicated in Table 2 (see legends). (middle) Variance of the vapor mixing ratio fluctuations vs time (s). (bottom) Variance of temperature fluctuations $\left(\mathrm{K}^{2}\right)$ vs time (s). 
eventually the TKE and temperature fluctuations continue to decay. The intermediate maximum is due to buoyancy forcing, which acts as an amplifier when its own amplitude is still large enough. When comparing $u_{i}^{2}\left(\mathbf{x}, t_{0}\right)$ with $u_{z}^{2}\left(\mathbf{x}, t_{0}\right)$, we observed that both quantities evolve in similar filaments for the period of the transient growth, that is, for a period that lasts for a few seconds starting after $4-5 \mathrm{~s}$. We interpret this finding as a clear indication that the initial buoyancy profile (as seen in Fig. 1c) amplifies vertical velocity fluctuations primarily. Since the whole system decays in the meantime, the intermediate peak in the fluctuations can be interpreted by the coupling of vertical velocity and buoyancy for the period at which temperature differences are still large enough. In turn, the transiently amplified vertical velocity fluctuations enhance the temperature fluctuations for this short period, which is displayed in Fig. 1c.

The amplitude of the transient growth increases with the amount of liquid water that is contained in the initial cloud slab. The middle panel of Fig. 2 displays the temporal evolution of the variance of the vapor mixing ratio fluctuations. The fluctuations are defined as

$$
q_{v}^{\prime}(\mathbf{x}, t)=q_{v}(\mathbf{x}, t)-\left\langle q_{v}(t)\right\rangle_{V},
$$

where we take the time-dependent volume mean correspondingly. The presence of the additional large-scale driving enhances the decay. We also observe that differences among the three runs in each of the two series remain small, implying that the feedback from the condensation rate source term in Eq. (5) remains very small. However, this difference is bigger in the stationary case than in the decaying case. The bottom panel of Fig. 2 displays the variance of the temperature field fluctuations, which have been calculated in the same way as those of the vapor mixing ratio [cf. Eq. (20)]. The transient growth depends now clearly on the flow regime and the initial amount of liquid water in the slab. With increasing initial radius $R_{0}$, as indicated in the legend, the transient growth increases in amplitude and time, a manifestation of the enhanced contribution from the source term in Eq. (4). The additional bulk forcing suppresses this effect significantly as can be seen when comparing both series. To summarize, the large-scale forcing $\mathbf{f}_{\mathrm{LS}}$ brings the whole system close to the passive mixing case that has been studied by Kumar et al. (2012, 2013).

In the runs with additional driving, the mixing is completed when the phase relaxation time is reached (see Table 2). The turbulence volume is uniformly mixed and turbulence remains in the statistically stationary regime. In the decaying case, the system remains in a transient that ends when the turbulence is completely faded away.

\section{b. Mixing diagrams}

For the rest of the paper we focus on the microphysical response during the mixing process. To analyze the evolution in the mixing diagrams, we divide the initial slab into subdomains as displayed in Fig. 3. The droplets seeded in the four subdomains are colored differently according to their initial locations, as shown in the top panel. The bottom panel visualizes the initial distortion of the cloud slab, with some droplets entering regions of environmental air and other droplets simply mixing within the cloud filament. The Lagrangian treatment of individual cloud droplets allows not only the bulk microphysical properties of the cloud to be investigated, but the evolution of the full droplet size distribution. This includes, for example, the possibility of strong or even complete evaporation of individual droplets that experience sudden exposure to dry environmental conditions during the early mixing transients.

Mixing diagrams allow the microphysical response to mixing to be viewed; that is, to what extent is a given reduction in liquid water content $W \propto n_{d}\left\langle r^{3}\right\rangle$ due to a reduction in $n_{d}$ versus $\left\langle r^{3}\right\rangle$ ? The inhomogeneous and homogeneous mixing lines plotted in the mixing diagram correspond to equilibrium, thermodynamic conditions under the two extremes for mixing. To what extent the actual cloud microphysical conditions correspond to equilibrium states is not obvious, however, because until the mixing is complete the system can be considered to be transient. We follow Andrejczuk et al. (2009) therefore and plot microphysical trajectories within the mixing diagram, so that the instantaneous temporal evolution of microphysical properties can be visualized. To define the droplet number density and mean radius, it is necessary to define a sample volume. We begin by dividing the volume into 16 equally sized subslabs that occupy the full width of the initial cloud filament and are arranged $4 \times 4$ in the $y$ and $z$ dimensions (as in Fig. 3, except each colored region there is further divided into four subvolumes). Microphysical trajectories for runs S3 and D3, that is, for initial droplet radii of $20 \mu \mathrm{m}$, are shown in the top panels of Fig. 4, and trajectories for runs S2 and D2, that is, for initial droplet radii of $15 \mu \mathrm{m}$, are shown in the bottom panels. A first observation is that the trajectories at least roughly tend to follow the homogeneous mixing line as opposed to the inhomogeneous mixing limit. In fact, while the individual trajectories may deviate during the transient behavior, the final points are quite close to the homogeneous mixing curve. This builds confidence in the notion that the Damköhler number, as defined here, indeed captures the essential behavior of the system. As summarized in Table $2, \mathrm{Da}_{\eta} \ll 1$ in all cases, so the 

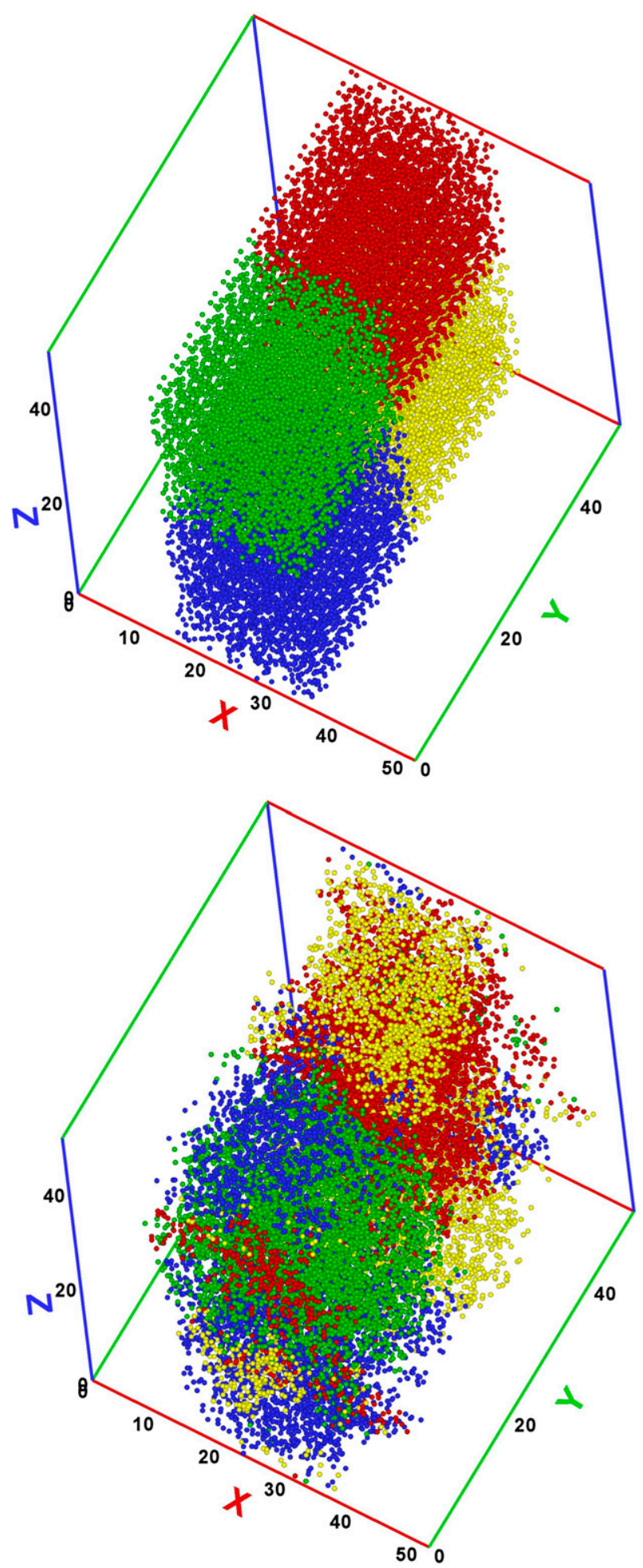

FIG. 3. Illustration of the Lagrangian mixing of the cloud water droplets. (top) Out of the 8.8 million droplets, we select 20000 droplets and color them differently based on their initial position in the cloud filament. (bottom) The mixing and entrainment has progressed to $1.25 \mathrm{~s}$ or $0.3 T_{L}$. This subdivision of the cloud filament will be also used later in the text when we discuss the mixing process in detail. dissipation-scale mixing is expected to be strongly in the homogeneous limit. Even for the large eddies, $\mathrm{Da}_{L} \sim 1$, and so the largest simulated scales are only expected to be in the transition range between homogeneous and inhomogeneous mixing. Indeed, a hint of this initially inhomogeneous behavior can be seen in the early transient response, in which the trajectories can be observed to initially follow the horizontal mixing line (see especially the bottom-left panel). Ultimately, a steady state is reached, and because the initial droplet radii are sufficiently large and the turbulent mixing is sufficiently rapid, few droplets are able to completely evaporate and the mean properties of the mixed-cloud approach the homogeneous limit. As would be expected, the trajectories from the runs with smaller initial radius progress farther down the homogeneous mixing lines compared to those with larger initial radius: a straightforward result of conservation of water mass, given the identical environmental conditions in the two cases.

Several differences are immediately evident when comparing the stationary forced results (Fig. 4, left) and the decaying results (Fig. 4, right). First, the variability between microphysical trajectories is significantly larger for the decaying compared to the stationary turbulence runs. Second, the endpoints of the trajectories of D3 and D2 do not reach as closely to the homogeneous mixing line, compared to S3 and S2. These observations can be interpreted to result directly from the strongly suppressed fluctuations in vapor and temperature fields (see middle and bottom panels of Fig. 2) in the stationary forced turbulence relative to the decaying turbulence. Qualitatively, the decaying turbulence dies out before the mixture has become thoroughly homogenized, allowing random fluctuations in temperature, vapor concentration, and droplet number density to persist and therefore for the resulting droplet radius fluctuations to become more pronounced. The scatter in the trajectory endpoints is especially noticeable in run $\mathrm{D} 2$, with $n_{d} / n_{d, 0}$ ranging from approximately 0.4 to 0.6 even in the final, mixed state. It is also intriguing that in the top-right panel, corresponding to run D3, several subvolumes achieve $n_{d} / n_{d, 0}>1$ during the initial mixing. In fact, in just a very short time, the trajectories span $n_{d} / n_{d, 0}$ of 0.4 1.1 in that case, eventually homogenizing to a range of $\Delta n_{d} / n_{d, 0} \approx 0.1$.

To investigate the possible influence of the sampling geometry on the results, we vary the subslab size and show the resulting trajectories in Fig. 5. For this comparison, only results for the decaying turbulence D1 are shown. Here, there is complete evaporation of the cloud when mixing is complete, but the trajectories show interesting differences. In the case with fewer, larger subvolumes (4 vs 16), there is considerably less variability. 

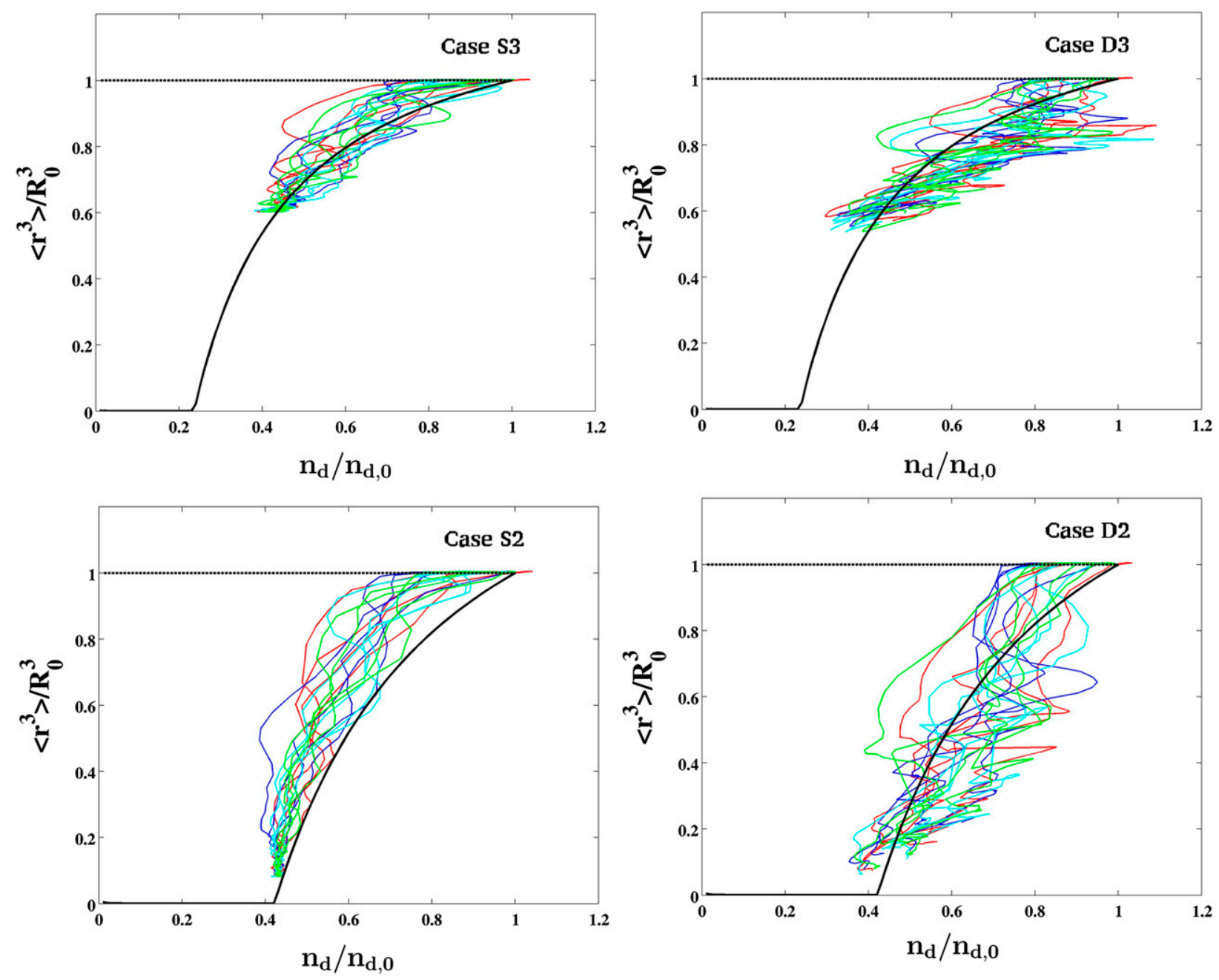

FIG. 4. Mixing diagrams. Mean cubic radius and mixture fraction have been calculated in 16 equally sized subslabs with $L_{x, 1} \leq x \leq L_{x, 2}$. They are obtained by splitting the original cloud filament. Details of the four displayed cases are given in Table 2.

This is not a surprise, since these coarser subvolumes are rather close to the large-eddy scale, whereas the smaller subvolumes lie in the inertial subrange. It should be noted that the very slight increases beyond $\left\langle r^{3}\right\rangle / R_{0}^{3}=1$ are a result of the small water vapor supersaturation in the initial profile of $q_{v}$ (cf. Fig. 1).

Especially interesting in the D1 run is the appearance of what can be termed an inhomogeneous offset: the curves appear quite similar to the shape of the homogeneous mixing curve but are shifted to smaller values of $n_{d} / n_{d, 0}$. This can be interpreted as resulting from the relative magnitude of $\tau_{\text {phase }}$ and the single-droplet $\tau_{\text {evap. }}$. Case D1 with $R_{0}=10 \mu \mathrm{m}$ is the only scenario in which $\tau_{\text {evap }}$ is significantly less than $\tau_{\text {phase }}$. Lehmann et al. (2009) showed that the relevant microphysical time scale describing the response to turbulent mixing is the smaller of $\tau_{\text {phase }}$ and $\tau_{\text {evap. }}$. That is because $\tau_{\text {phase }}$ is calculated by assuming constant $R$, whereas $\tau_{\text {evap }}$ is calculated by assuming constant $S$, neither strictly correct, but the smaller of two time scales indicating which assumption is more accurate. Defining a Damköhler number based on $\tau_{\text {evap }}$ results in $\mathrm{Da}_{L \text {,evap }}=4.4$ for cases $\mathrm{S} 1$ and D1, significantly greater than unity and therefore favoring inhomogeneous mixing at the largest scales in the inertial subrange. This can be quantified via the transition length scale, defined as the length within the inertial subrange at which $\mathrm{Da}=1$, that is, $l^{*}=\left(\tau_{\text {evap }}^{3}\langle\varepsilon\rangle\right)^{1 / 2}$. For case S1 and D1, we obtain $l^{*}=$ $5 \mathrm{~cm}$, which is a factor of 10 smaller than the large-eddy scale $L_{x}$ and a factor of 50 greater than the Kolmogorov length scale $\eta_{K}$ (see Table 1). The trajectories shown in Fig. 5 therefore can be taken as direct demonstration of the concept of a shift from inhomogeneous to homogeneous mixing as mixing proceeds from the energy injection scale $L_{x}$, through $l^{*}$, and ultimately down to the energy dissipation scale $\eta_{K}$. It should be noted that this 

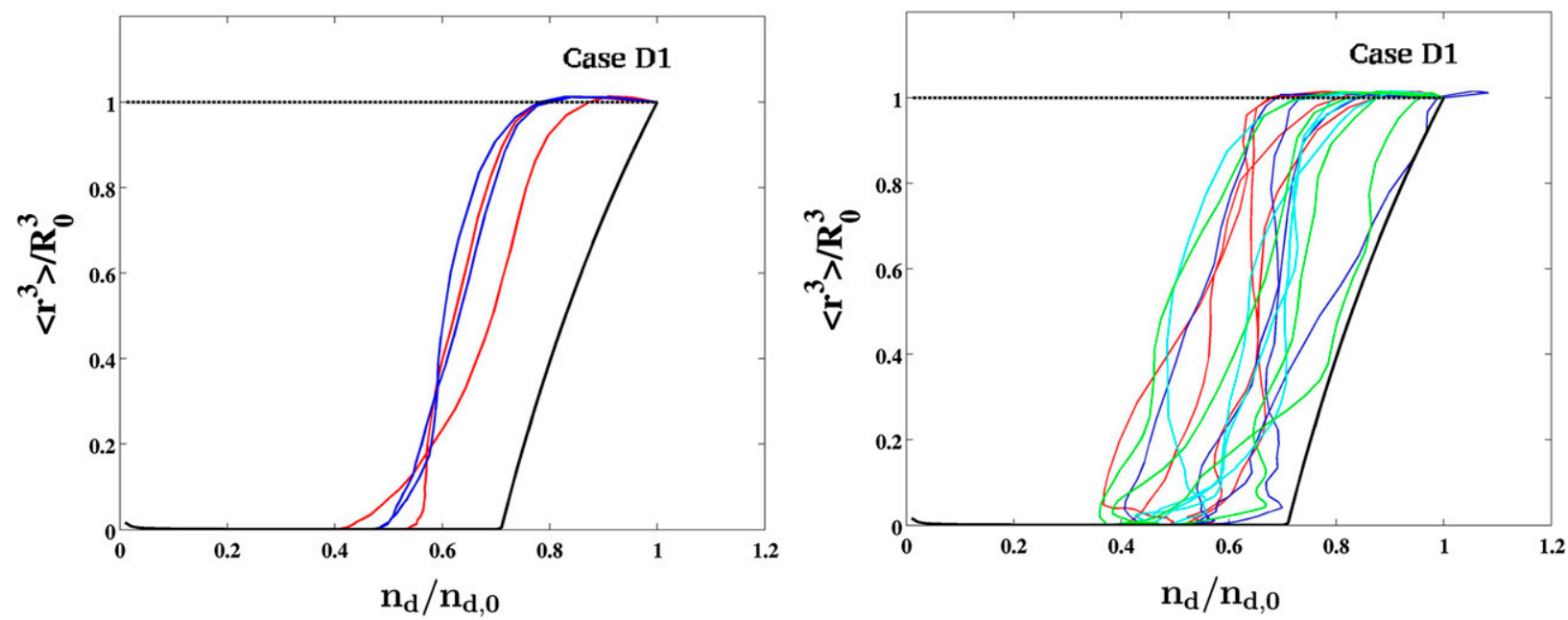

FIG. 5. Mixing diagrams. Mean cubic radius and mixture fraction have been calculated in (left) 16 and (right) 4 equally sized subslabs with $L_{x, 1} \leq x \leq L_{x, 2}$. They are obtained by splitting the original cloud filament. Both figures are for case D1 as indicated in Table 2.

adds support to the parameterization concept of Lu et al. (2013), which is based on the notion of the transition length scale. Finally, Fig. 5 and its interpretation also provide a response to the concluding challenge posed by Burnet and Brenguier (2007, p. 2009): “A challenge for such numerical simulation will be to replicate the typical features seen in the $N-D_{v}^{3}$ diagrams...with homogeneous-like mixing features at high LWC dilution ratio, progressively moving toward an inhomogeneouslike mixing process when the dilution ratio decreases." We note, finally, that there is a hint of an inhomogeneous offset in the mixing diagram for case S2 in Fig. 4; we speculate that the offset is consistent with the fact that $\tau_{\text {evap }}$ is slightly less than $\tau_{\text {phase }}$, and $\mathrm{Da}_{L \text {,evap }}=$ 2.0 , with the result that partial evaporation can occur. The absence of this signature for case D2 is not understood at this time.

A wide range of variability in the shape of the individual trajectories is evident and has implications for the interpretation of field measurements. Even without the difficulties of measurement limitations and uncertainties, considerable scatter in microphysical quantities can be expected simply as a result of the inevitability of sampling cloud mixing events during a range of transient times. Most field measurements displayed on mixing diagrams [e.g., in Burnet and Brenguier (2007), Gerber et al. (2008), or Lehmann et al. (2009)] have been interpreted, at least implicitly, as following homogeneous or inhomogeneous mixing curves that correspond to thermodynamic equilibrium states. The trajectories in Figs. 4 and 5 clearly show, however, that even when the Damköhler number favors homogeneous mixing, a wide range of $n_{d}-\left\langle r^{3}\right\rangle$ values both below and above the homogeneous mixing curve are encountered. Figure 5 further suggests that the measurement volume geometry may also influence the variability in the measured $n_{d}-\left\langle r^{3}\right\rangle$ values, with the apparent conflict between the desire to reduce the measurement volume in order to resolve finescale mixing features, but at the same time realizing that finer volumes lead to greater uncertainty in comparing to the theoretical mixing predictions.

\section{c. Distributions of droplet size and supersaturation}

In this section, we consider the microphysical response of the system in more detail by looking at droplet size distributions, including the fraction of fully evaporated droplets, and probability density functions for supersaturation along Lagrangian droplet paths. Since the droplet radii remain at $r \leq 20 \mu \mathrm{m}$ in our system, effects of droplet inertia, for example, the so-called sling effect (Falkovich and Pumir 2007; Bewley et al. 2013), are subdominant. The typical order of magnitude of the Stokes number $\mathrm{St}_{\eta}=\tau_{p} / \tau_{\eta}<10^{-1}$, as discussed in Kumar et al. (2013).

Some aspects of the conceptual picture that has emerged from the mixing diagrams can be seen from a different perspective by considering the total number of droplets versus time, as shown in Fig. 6. For both S1 and D1, all droplets eventually evaporate because there is insufficient condensed water to bring the full mixture to saturation. The cloud with forced turbulence fully evaporates within approximately two large-eddy times, whereas the cloud with decaying turbulence requires more than four large-eddy times. This is a result, on the one hand, of the forced turbulence more rapidly and thoroughly mixing cloudy and clear air. The decaying turbulence, on the other hand, presumably leaves 


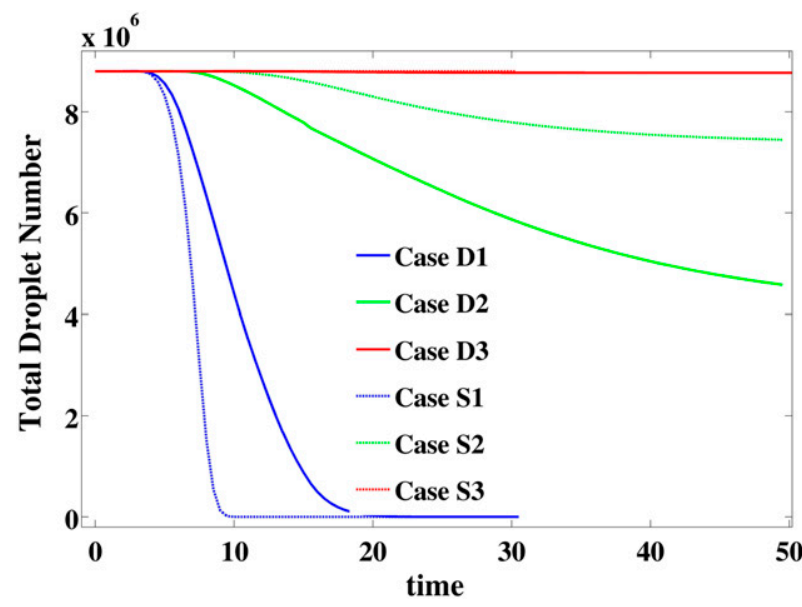

FIG. 6. Total number of droplets in the computational domain vs time for all six cases. For cases S1 and D1, the equilibrium state has a liquid water content of zero. The rate at which droplets evaporate is higher for the forced turbulence compared to the decaying turbulence. All other cases end with finite liquid water contents after transients have vanished; cases S3 and D3 show little full evaporation, but S2 and D2 have significant loss of droplets. In those intermediate cases, the number of fully evaporated droplets is greater for the decaying turbulence. These contrasting results are interpreted in the text. The time is measured in seconds.

pockets of cloud and clear air that dissipate partially through diffusion and gravitational settling as the turbulence weakens. Interestingly, even with the single droplet evaporation time $\tau_{\text {evap }}=0.9 \mathrm{~s}$ being approximately $1 / 4$ of the large-eddy time $T_{L}$, significant disappearance of droplets does not occur until $t>T_{L}$. For case D1, which was illustrated in Fig. 5 and exhibited a significant inhomogeneous offset, the time scale for the transition from inhomogeneous to homogeneous mixing can be seen to approximately two large-eddy times.

In the other extreme, cases S3 and D3, essentially no droplets experience full evaporation, again confirming that the relevant time scale is the lesser of $\tau_{\text {phase }}$, and $\tau_{\text {evap }}$ determines the mixing response, that is, phase relaxation in both of these cases. The intermediate cases S2 and D2 are interesting: although the conservation of water mass constraint allows for finite liquid water content after equilibrium is reached, a significant fraction of the droplets fully evaporate during the transient response. In contrast to the fully evaporating cloud cases (S1 and D1), here the forced turbulence leads to a reduction in the number of completely evaporated droplets compared to the decaying turbulence. In this case, the opposite response is somewhat paradoxically attributed to the same cause: the delayed and somewhat incomplete nature of the mixing process in the case with decaying turbulence. The longer-lived inhomogeneities in the mixture provide droplets at the edge of or even inside of clear patches to experience strong evaporation for longer times. The contrasting results therefore have a similar explanation: decaying turbulence leaves longerlived patchiness in the mixing and therefore less direct approach to the equilibrium state. When the equilibrium state corresponds to fully evaporated cloud, certain droplets are in patches that take longer to reach that state; when the equilibrium state corresponds to a partially evaporated cloud, certain droplets are exposed to transient conditions for longer times and therefore do not survive.

Mean droplet properties are represented in the mixing diagrams discussed in section $3 \mathrm{~b}$, and now we look at the detailed droplet response to mixing by plotting probability density functions for droplet radius. Probability density functions (PDFs) are displayed in Fig. 7 for times $t=0.5$, $1.0,2.0,5.0,10.0,20.0$, and $30.0 \mathrm{~s}$ for the two extreme liquid water contents: simulations S1 and D1 in the top row and S3 and D3 in the bottom row. The two top panels correspond to the largest initial Damköhler number, with $\mathrm{Da}_{L \text {,evap }}=4.4$, and therefore the most inhomogeneous response of the size distribution. The two bottom panels correspond to a scenario in which complete droplet evaporation does not occur and for which the large-eddy Damköhler numbers are close to unity. The size PDFs indeed exhibit certain features observed for extreme limits of inhomogeneous and homogeneous mixing, as explored by Kumar et al. (2012). That those trends still hold is significant because in that study the temperature field was neglected; this therefore supports the conclusion that buoyancy effects are relatively minor in determining the mixing at these small scales, at least for the conditions studied here. The S3 simulation case is the most characteristic of homogeneous mixing, with the size distribution broadening somewhat during the early mixing (within approximately the first two large-eddy times) and then evolving mostly via the shifting of the narrow distribution to smaller mean radius values. Cases S1 and D1 both exhibit characteristic features of inhomogeneous mixing: the rapid appearance of a negatively skewed distribution, with the negative tail being approximately exponential. Although the tail is pronounced, it does not strongly affect the mean volume droplet radius. Only after approximately one large-eddy time does the main droplet size distribution mode shift to smaller radius values, consistent with the initially inhomogeneous and subsequently homogeneous mixing behavior noted in the mixing diagrams (cf. Fig. 5). This again substantiates the transition from inhomogeneous to homogeneous mixing as occurring on a time scale of approximately $2 T_{L}$.

Droplet growth is directly coupled to the local temperature and vapor mixing ratio fields via the water vapor supersaturation, so we can gain further understanding by plotting probability density functions for $S$, 

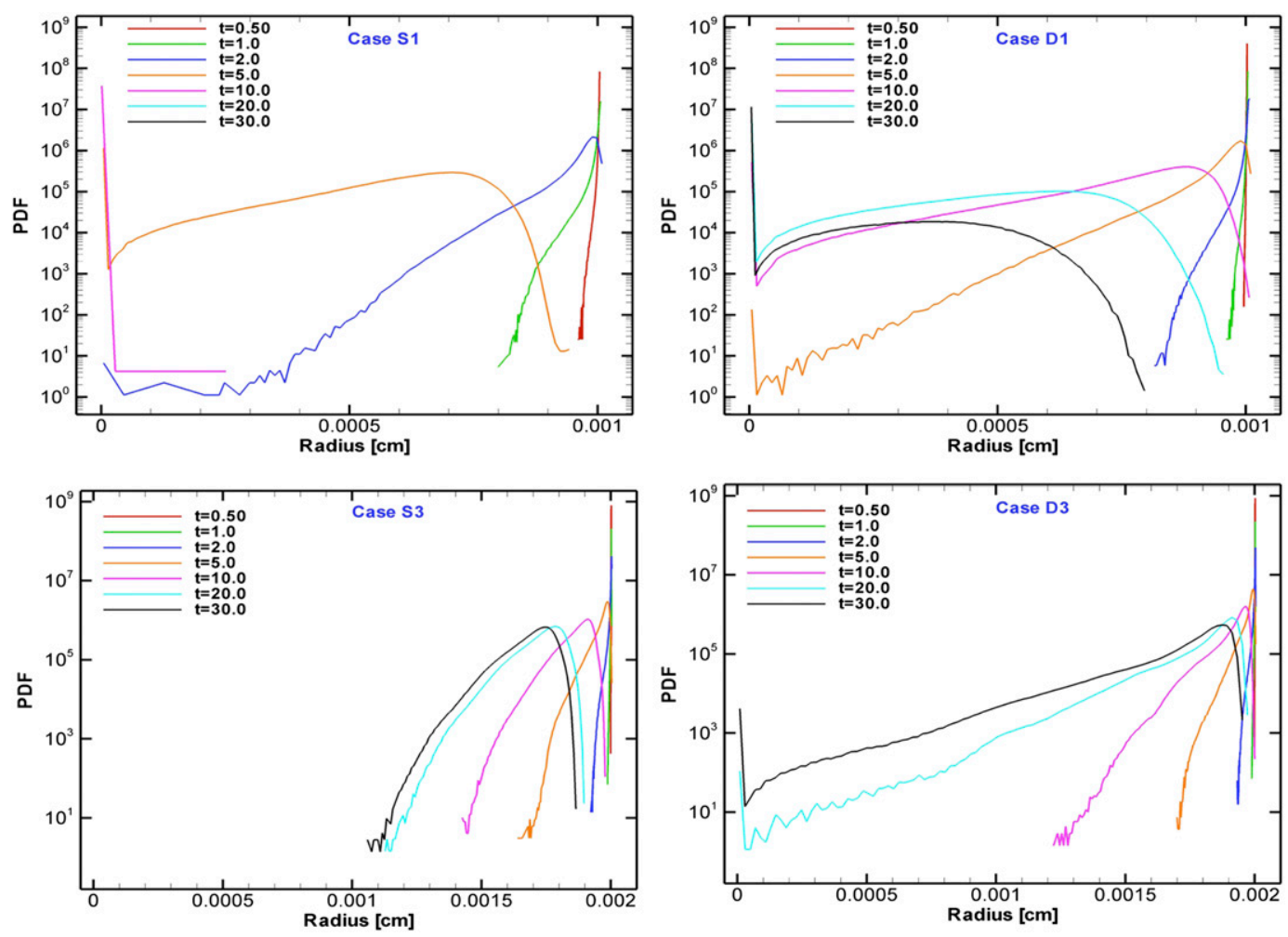

FIG. 7. PDFs of the size distribution at different times (see legend). (top left) Case S1 and (top right) case D1. (bottom left) Case S3 and (bottom right) case D3 (see Table 2). The two extreme cases are shown in order to illustrate microphysical response under conditions favoring inhomogeneous response in the top row and homogeneous response in the bottom row.

as sampled along Lagrangian droplet paths. The PDFs are displayed in Fig. 8 for the same times and simulation cases as in Fig. 7. Indeed, as noted by Kumar et al. (2012), there is always a rapid formation of a negative exponential tail, likely related to the well-known intermittent properties of scalar mixing in turbulence. The appearance of a negative exponential tail in the droplet size distribution observed in Fig. 7 results directly from the similar shape in the supersaturation PDFs. For case S3, however, it should be noted that although the supersaturation PDF displays a negative exponential tail, the droplet size distribution does not because of the rapid and thorough (homogeneous) mixing and the associated collapse of the negative supersaturation tail within several large-eddy times. As expected, the supersaturation PDF in cases S3 and D3 eventually approaches a delta function at $S=0$. In cases $\mathrm{S} 1$ and $\mathrm{D} 1$, the supersaturation peak never recovers to $S=0$ due to insufficient initial liquid water content.

Compared to the others, the size distributions for simulation case D3 are somewhat enigmatic. Although the initial $\mathrm{Da}$ are identical to $\mathrm{S} 3$, the droplet size distributions show much more inhomogeneous-like mixing behavior, that is, the formation of a pronounced negative exponential tail. Despite the tail formation, however, the mean droplet radius does not change significantly, unlike for cases S1 and D1. The negative tail formation is interpreted to result from the persistence of cloudclear air gradients beyond the typical times of $t \sim T_{L}$. Thus, although the initial $\mathrm{Da}_{L}$ would suggest rapid mixing and dissipation of gradients, in fact some of the gradients end up essentially frozen in as the turbulent kinetic energy collapses. Similarly, in case D1, it can be seen that the supersaturation PDF remains quite broad even for $t \gg T_{L}$, because turbulent mixing has become very inefficient. This implies that in these cases the buoyancy feedback is inadequate to force significant subsequent mixing. There is little positive feedback due to buoyancy effects in the mixing process, even for the very dry environment and large liquid water contents considered here.

\section{Summary and conclusions}

The work described here is focused on simulating the mixing of a cloudy filament with environmental air, under conditions when collisions are not relevant. Although the initial configuration (slab cloud) is highly idealized, we take the liberty in our numerical experiments to study this mixing problem under controlled conditions 

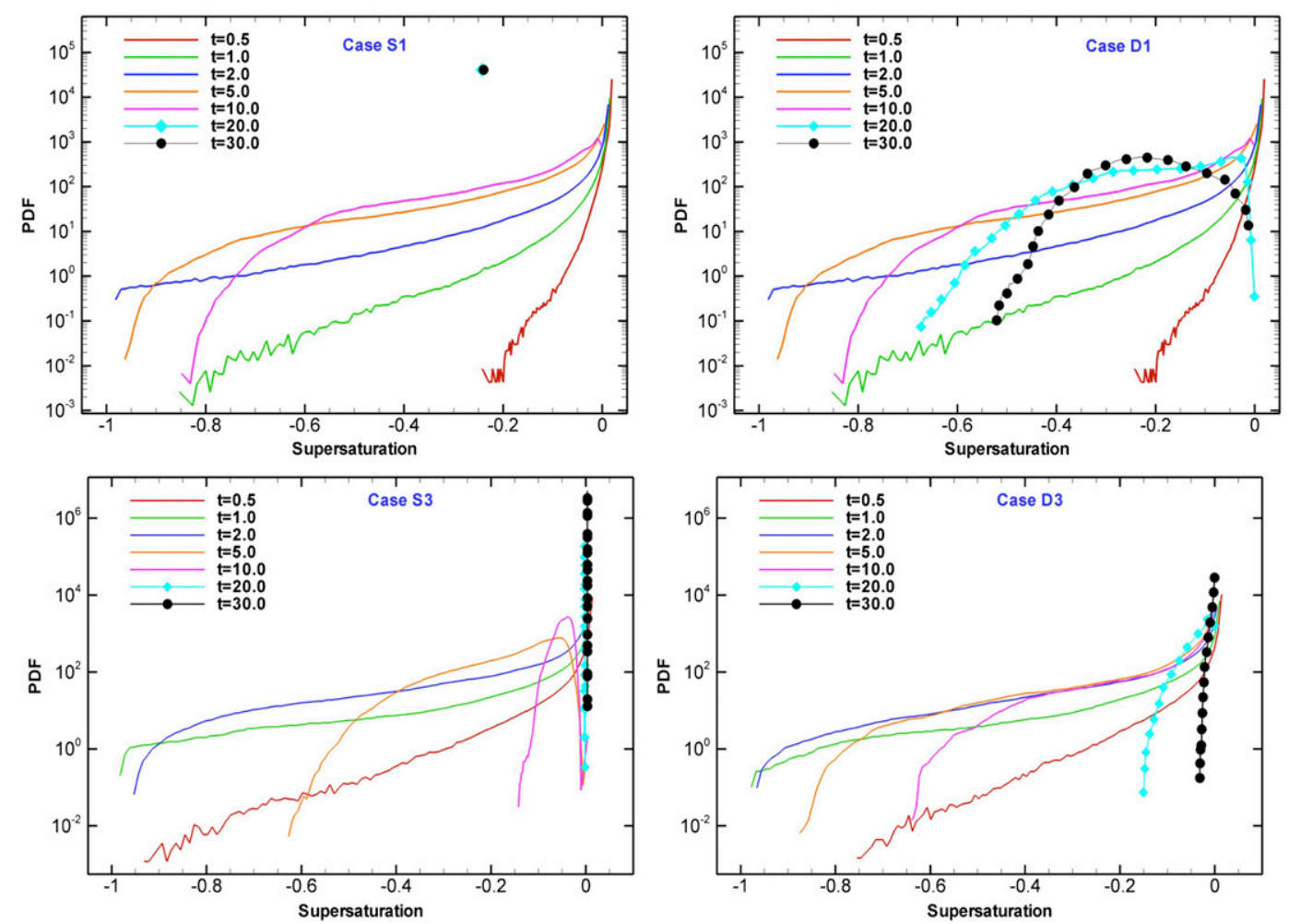

FIG. 8. PDFs of the supersaturation along the Lagrangian droplet trajectories at different times (see legend). (top left) Case S1 and (top right) case D1. (bottom left) Case S3 and (bottom right) case D3 (Table 2). The two extreme cases are shown in order to illustrate microphysical response under conditions favoring inhomogeneous response in the top row and homogeneous response in the bottom row. Data are for the same cases and times as in Fig. 7.

that are very difficult to find in cloud measurements. We have a clearly defined cloud-clear air interface and can study essential input parameters disentangled from each other, for example, initial water mass (controlled by $R_{0}$ ) and turbulence conditions. The approach captures the full complexity of the microphysical response to a mixing event, including Eulerian description of velocity, temperature, and water vapor density fields and Lagrangian description of the cloud droplet population. Cloud droplet growth and evaporation is coupled to the water vapor field, so that the response to local fluctuations is captured, and the droplet size distribution evolves in response to the turbulent mixing. Therefore, all scales from approximately $50 \mathrm{~cm}$ and below are resolved, free of any parameterization.

The microphysical response to mixing is represented via trajectories (i.e., time histories) within an $n_{d}-\left\langle r^{3}\right\rangle$ space. As pioneered by Jensen et al. (1985), Burnet and Brenguier (2007), Andrejczuk et al. (2009), and others, this approach allows the relative contributions of droplet number density and droplet radius to the liquid water content, $W \propto n_{d}\left\langle r^{3}\right\rangle$. The trajectories generally show agreement with the theoretical homogeneous mixing curve when $\mathrm{Da}_{L} \leq 1$. There are significant deviations, however, both above and below the homogeneous mixing line, as a result of turbulent fluctuations. These fluctuations arising from the transient response to turbulent mixing pose a challenge to the interpretation of in situ measurements. The magnitude of the fluctuations also depends on the averaging volume, with variability increasing as the characteristic averaging length scale reduces below the large-eddy length scale.

When turbulent mixing is externally forced so that the energy dissipation rate is stationary, relaxation to the mixed state is faster and the fluctuations in $n_{d}-\left\langle r^{3}\right\rangle$ space are small relative to those occurring when the turbulence is decaying except for the buoyancy feedback. For the decaying turbulence case, trajectories do not always end on the homogeneous mixing line within the simulated times, but for the forced turbulence case, all trajectories converge to that thermodynamic equilibrium state. In terms of the scalar fields, the process is always found to be strongly time dependent. The scalar fields basically decay quickly, whether the flow is statistically stationary or not. Their feedback on the flow via the buoyancy term is found to be weak; in the decaying convective regime (D runs), the velocity is very 
close to freely decaying turbulence. Furthermore, and for this reason as well, the $\mathrm{S}$ runs are very close to the passive mixing problem that was studied by Kumar et al. (2012, 2013).

The results confirm the finding of Lehmann et al. (2009) that when the time scale for the evaporation of a single droplet in the environmental air is less than the phase relaxation time scale, it becomes the governing factor in determining the Damköhler number. There has been longstanding disagreement in the literature as to whether the phase relaxation or droplet evaporation time should be considered as the relevant microphysical time scale, so although more of the parameter space should be investigated, this adds clarity to the picture.

One of the simulated cases has a large-eddy Damköhler number $\mathrm{Da}_{L}$ significantly greater than unity, which is typically a range that is challenging to achieve in a direct numerical simulation (DNS). The trajectories in $n_{d}-\left\langle r^{3}\right\rangle$ space for that case show a distinct shape, with initial inhomogeneous mixing eventually changing to follow the shape of a homogeneous mixing curve, but they are shifted to smaller $n_{d}$. This transition from inhomogeneous to homogeneous mixing corroborates the concept of the transition length scale, that is, the length scale within the inertial subrange at which $\mathrm{Da}=1$, above which mixing is primarily inhomogeneous and below which mixing is primarily homogeneous (Lehmann et al. 2009). This leads to the observed "inhomogeneous offset" in the $n_{d}-\left\langle r^{3}\right\rangle$ trajectories. Further studies under a variety of conditions with $\mathrm{Da}_{L}>1$ and with the transition length scale set to various stages within the inertial subrange are needed in order to guide quantitative understanding of the inhomogeneous offset.

Droplet radius and Lagrangian-sampled supersaturation PDFs show additional details about the microphysical response to mixing. In all cases, there is a sudden and rapid appearance of a negative exponential tail in the supersaturation PDF, presumably resulting from the initial multiscale mixing of the scalar (temperature and vapor density) fields. In the more homogeneous cases, the supersaturation tail quickly collapses and the droplet size distribution has relatively little time to broaden as a result. Generally, the size distribution shifts slowly to a smaller mean radius as all droplets respond to the well-mixed, homogeneous background. In the inhomogeneous mixing cases, such as S1 and D1, the droplet size distributions have sufficient time to adjust to the skewed supersaturation PDFs and therefore form their own negative exponential tails. The mean droplet radius, however, remains relatively constant until the transition to homogeneous mixing occurs, and the distribution mode shifts to smaller sizes.

The simulations reported here are highly idealized and only cover a portion of the large microphysical and thermodynamic parameter space for realistic clouds. The thermodynamic conditions of this study were taken to be similar to the cloud observations reported by Lehmann et al. (2009), which were made near the freezing point of water. In subsequent work, we aim to consider a wider range of the parameter space, including cases allowing us to investigate the role played by buoyancy as the temperature is increased and latent heating effects become more pronounced. Microphysically, the clouds considered here are in the extreme of dry environment, high droplet concentration, and large droplet diameters (high liquid water content) so as to reach large Damköhler numbers. In the future, we will extend the study to conditions more applicable to stratocumulus, that is, with lower cloud droplet number densities and smaller droplet diameters, and to small cumulus in more humid environments. In general, lower liquid water contents tend toward larger phase relaxation times and therefore smaller Damköhler numbers and, all else being equal, more homogeneous mixing. It can be expected, in contrast, that more humid environments will tend to favor more inhomogeneous mixing. Finally, the possible role of a gradient in turbulence intensity, from relatively high inside the cloud filament to relatively low in the clear air as is commonly observed in cloud measurements (e.g., Siebert et al. 2013), will be investigated.

The study raises several questions that will require further study to answer. What is the origin of the negative exponential tail in the supersaturation PDFs and can they be quantitatively tied to the distortion of the cloud-clear air interface through turbulent mixing? What determines, again quantitatively, the magnitude of the inhomogeneous offset such as observed in Fig. 5? Presumably, the dilution and reduction in $n_{d}$ progresses until the mixing cascades down to the transition length scale $l^{*}$, at which point droplets start to see a more uniform background and evaporate in unison. Finally, and perhaps most importantly, how will the nature of mixing change as the limit $\mathrm{Da}_{L} \gg 1$ is reached, as is expected in natural clouds? This is ultimately what will need to be understood in order to develop physically based parameterizations of the microphysical response to mixing across the turbulent cascade. Extending simulations such as those performed here to progressively higher Reynolds numbers, and therefore a larger range of turbulent length scales, will allow the phenomenon to be explored with the level of detail enabled by fully Lagrangian droplet representation. 
Acknowledgments. This work was supported by the Deutsche Forschungsgemeinschaft within the Priority Program Metström and by U.S. National Science Foundation Grant AGS-1026123. Computations were supported by the John von Neumann Institute for Computing under Grant HIL03 at the Jülich Supercomputing Centre in Germany.

\section{REFERENCES}

Andrejczuk, M., W. W. Grabowski, S. P. Malinowski, and P. K. Smolarkiewicz, 2004: Numerical simulation of cloud-clear air interfacial mixing. J. Atmos. Sci., 61, 1726-1739, doi:10.1175/ 1520-0469(2004)061<1726:NSOCAI >2.0.CO;2.

,,--- , and,- 2006 : Numerical simulation on cloudclear air interfacial mixing: Effects on cloud microphysics. J. Atmos. Sci., 63, 3204-3225, doi:10.1175/JAS3813.1.

,,--- , and - 2009: Numerical simulation of cloudclear air interfacial mixing: Homogeneous versus inhomogeneous mixing. J. Atmos. Sci., 66, 2493-2500, doi:10.1175/ 2009JAS2956.1.

Baker, M. B., R. G. Corbin, and J. Latham, 1980: The influence of entrainment on the evolution of cloud droplet spectra: I. A model of inhomogeneous mixing. Quart. J. Roy. Meteor. Soc., 106, 581-598, doi:10.1002/qj.49710644914.

— , R. E. Breidenthal, T. W. Choularton, and J. Latham, 1984: The effects of turbulent mixing in clouds. J. Atmos. Sci., 41, 299-304, doi:10.1175/1520-0469(1984)041<0299:TEOTMI>2.0.CO;2.

Bewley, G. P., E.-W. Saw, and E. Bodenschatz, 2013: Observation of the sling effect. New J. Phys., 15, 083051, doi:10.1088/ 1367-2630/15/8/083051.

Broadwell, J. E., and R. E. Breidenthal, 1982: A simple model of mixing and chemical reaction in a turbulent shear. J. Fluid Mech., 125, 397-410, doi:10.1017/S0022112082003401.

Burnet, F., and J.-L. Brenguier, 2007: Observational study of the entrainment-mixing process in warm convective clouds. J. Atmos. Sci., 64, 1995-2011, doi:10.1175/JAS3928.1.

Calzavarini, E., C. R. Doering, J. D. Gibbon, D. Lohse, A. Tanabe, and F. Toschi, 2006: Exponentially growing solutions in homogeneous Rayleigh-Bénard convection. Phys. Rev. E, 73, 035301(R), doi:10.1103/PhysRevE.73.035301.

de Lozar, A., and J. P. Mellado, 2014: Cloud droplets in a bulk formulation and its application for the buoyancy reversal instability. Quart. J. Roy. Meteor. Soc., doi:10.1002/qj.2234, in press.

de Rooy, W. C., and Coauthors, 2013: Entrainment and detrainment in cumulus convection: An overview. Quart. J. Roy. Meteor. Soc., 139, 1-19, doi:10.1002/qj.1959.

Falkovich, G., and A. Pumir, 2007: Sling effect in collisions of water droplets in turbulent clouds. J. Atmos. Sci., 64, 4497-4505, doi:10.1175/2007JAS2371.1.

Ferziger, J. H., and M. Perić, 2001: Computational Methods in Fluid Dynamics. Springer, 423 pp.

Gerber, H. E., G. M. Frick, J. B. Jensen, and J. G. Hudson, 2008: Entrainment, mixing, and microphysics in trade-wind cumulus. J. Meteor. Soc. Japan, 86A, 87-106, doi:10.2151/jmsj.86A.87.

Grabowski, W. W., 2007: Representation of turbulent mixing and buoyancy reversal in bulk cloud models. J. Atmos. Sci., 64, 3666-3680, doi:10.1175/JAS4047.1.

Heus, T., and H. J. J. Jonker, 2008: Subsiding shells around shallow cumulus clouds. J. Atmos. Sci., 65, 1003-1018, doi:10.1175/ 2007JAS2322.1.
Jensen, J. B., and M. B. Baker, 1989: A simple model of droplet spectral evolution during turbulent mixing. J. Atmos. Sci., 46, 2812-2829, doi:10.1175/1520-0469(1989)046<2812: ASMODS $>2.0$. CO;2.

— - P. H. Austin, M. B. Baker, and A. M. Blyth, 1985: Turbulent mixing, spectral evolution and dynamics in warm cumulus cloud. J. Atmos. Sci., 42, 173-192, doi:10.1175/ 1520-0469(1985)042<0173:TMSEAD>2.0.CO;2.

Kostinski, A. B., 2009: Simple approximations for condensational growth. Environ. Res. Lett., 4, 015005, doi:10.1088/ 1748-9326/4/1/015005.

Krueger, S. K., C.-W. Su, and P. A. Murtry, 1997: Modeling entrainment and finescale mixing in cumulus clouds. J. Atmos. Sci., 54, 2697-2712, doi:10.1175/1520-0469(1997)054<2697: MEAFMI > 2.0.CO;2.

Kumar, B., F. Janetzko, J. Schumacher, and R. A. Shaw, 2012: Extreme responses of a coupled scalar-particle system during turbulent mixing. New J. Phys., 14, 115020, doi:10.1088/ 1367-2630/14/11/115020.

_ J. Schumacher, and R. A. Shaw, 2013: Cloud microphysical effects of turbulent mixing and entrainment. Theor. Comput. Fluid Dyn., 27, 361-376, doi:10.1007/s00162-012-0272-z.

Lanotte, A. S., A. Seminara, and F. Toschi, 2009: Cloud droplet growth by condensation in homogeneous isotropic turbulence. J. Atmos. Sci., 66, 1685-1697, doi:10.1175/2008JAS2864.1.

Latham, J., and R. L. Reed, 1977: Laboratory studies of the effects of mixing on the evolution of cloud droplet spectra. Quart. J. Roy. Meteor. Soc., 103, 297-306, doi:10.1002/ qj. 49710343607.

Lehmann, K., H. Siebert, and R. A. Shaw, 2009: Homogeneous and inhomogeneous mixing in cumulus clouds: Dependence on local turbulence structure. J. Atmos. Sci., 66, 3641-3659, doi:10.1175/2009JAS3012.1.

Lu, C., Y. Liu, and S. Niu, 2011: Examination of turbulent entrainment-mixing mechanisms using a combined approach. $J$. Geophys. Res., 116, D20207, doi:10.1029/2011JD015944.

,,,--- S. K. Krueger, and T. Wagner, 2013: Exploring parameterization for turbulent entrainment-mixing processes in clouds. J. Geophys. Res. Atmos., 118, 185-194, doi:10.1029/ 2012JD018464.

Malinowski, S. P., and I. Zawadzki, 1993: On the surface of clouds. J. Atmos. Sci., 50, 5-13, doi:10.1175/1520-0469(1993)050<0005: OTSOC $>2.0 . \mathrm{CO} ; 2$.

—, M. Andrejczuk, W. W. Grabowski, P. Korczyk, T. A. Kowalewski, and P. K. Smolarkiewicz, 2008: Laboratory and modeling studies of cloud-clear air interfacial mixing: Anisotropy of small-scale turbulence due to evaporative cooling. New J. Phys., 10, 075020, doi:10.1088/ 1367-2630/10/7/075020.

Rogers, R. R., and M. K. Yau, 1989: A Short Course in Cloud Physics. Pergamon Press, 293 pp.

Schumacher, J., K. R. Sreenivasan, and V. Yakhot, 2007: Asymptotic exponents from low-Reynolds-number flows. New J. Phys., 9, 89, doi:10.1088/1367-2630/9/4/089.

Siebert, H., and Coauthors, 2013: The fine-scale structure of the trade wind cumuli over Barbados: An introduction to the CARRIBA project. Atmos. Chem. Phys., 13, 10 061-10077, doi:10.5194/acp-13-10061-2013.

Spyksma, K., P. Bartello, and M. K. Yau, 2006: A Boussinesq moist turbulence model. J. Turbul., 7 (32), 1-24, doi:10.1080/ 14685240600577865.

Sreenivasan, K. R., R. Ramshankar, and C. Meneveau, 1989: Mixing, entrainment and fractal dimension of surfaces in 
turbulent flow. Proc. Roy. Soc. London, 421, 79-108, doi:10.1098/rspa.1989.0004.

Su, C.-W., S. K. Krueger, P. A. McMurtry, and P. H. Austin, 1998: Linear eddy modeling of droplet spectra evolution during entrainment and mixing in cumulus clouds. Atmos. Res., 4748, 41-58, doi:10.1016/S0169-8095(98)00039-8.

Vaillancourt, P. A., M. K. Yau, and W. W. Grabowski, 2001: Microscopic approach to cloud droplet growth by condensation.
Part I: Model description and results without turbulence. J. Atmos. Sci., 58, 1945-1964, doi:10.1175/1520-0469(2001)058<1945: MATCDG $>2.0 . C O ; 2$.

, — , P. Bartello, and W. W. Grabowski, 2002: Microscopic approach to cloud droplet growth by condensation. Part II: Turbulence, clustering, and condensational growth. J. Atmos. Sci., 59, 3421-3435, doi:10.1175/1520-0469(2002)059<3421: MATCDG $>2.0 . C O ; 2$. 